\title{
Density sensitivity of empirical functionals
}

\author{
Suhwan Song ${ }^{a}, \mathrm{Stefan} \mathrm{Vuckovic}^{b}, \mathrm{Eunji}_{\mathrm{Sim}}^{a, *}$, And Kieron Burke ${ }^{b}$ \\ ${ }^{a}$ Department of Chemistry, Yonsei University, 50 Yonsei-ro Seodaemun-gu, Seoul 03722, Korea \\ ${ }^{b}$ Departments of Chemistry and of Physics, University of California, Irvine, CA 92697, USA
}

December 2, 2020

\begin{abstract}
Empirical fitting of parameters in approximate density functionals is common. Such fits conflate errors in the self-consistent density with errors in the energy functional, but density-corrected DFT (DC-DFT) separates these two. We illustrate with catastrophic failures of a toy functional applied to $\mathrm{H}_{2}^{+}$at varying bond lengths, where the standard fitting procedure misses the exact functional; Grimme's D3 fit to noncovalent interactions, which can be contaminated by large density errors such as in the WATER27 and B30 datasets; and double-hybrids trained on self-consistent densities, which can perform poorly on systems with density-driven errors. In these cases, more accurate results are found at no additional cost, by using Hartree-Fock (HF) densities instead of self-consistent densities. For binding energies of small water clusters, errors are greatly reduced. Range-separated hybrids with 100\% HF at large distances suffer much less from this effect.
\end{abstract}

For the last quarter century, fitting of empirical parameters in approximate exchange-correlation functionals has been popular, especially given the early successes of Becke88 exchange, ${ }^{1}$ LeeYang-Parr correlation, ${ }^{2}$ and the global hybrid ideas of Becke, ${ }^{3}$ ultimately leading to the hugely successful B3LYP. ${ }^{4}$ Since then, the number of functionals and the number of parameters has proliferated, ${ }^{5,6}$ and often dozens of parameters are fitted to dozens of databases, with thousands of benchmark data.

There are many pitfalls to such fitting, but we focus on just one. This danger is unambiguous, has nothing to do with choices of parameters or datasets, and entirely avoidable. Almost all such fittings consist of running one or more selfconsistent DFT calculations, evaluating an energy difference, and comparing it with a (presumably accurate) energy from the database. (In the case of bond lengths, the difference is an infinitesimal, determining where an energy derivative vanishes). The accuracy of self-consistent densities was recently highlighted, ${ }^{7}$ and how errors in the density can be related to errors in the energy. ${ }^{8-20}$

Background: The theory of density-corrected DFT (DCDFT) has been developed over the past decade. ${ }^{12}$ Whenever a self-consistent (SC) DFT calculation is run, there are two distinct sources of error. The total error of such calculations is $\Delta E=\tilde{E}[\tilde{n}]-E[n]$, where $E$ and $n$ are the exact energy functional and density, and $\tilde{E}$ and $\tilde{n}$ are their approximate counterparts. We decompose $\Delta E$ as ${ }^{14,19,21}$ :

$$
\Delta E=\underbrace{\tilde{E}[\tilde{n}]-\tilde{E}[n]}_{\Delta E_{\mathrm{D}}}+\underbrace{\tilde{E}[n]-E[n]}_{\Delta E_{\mathrm{F}}} .
$$

where $\Delta E_{\mathrm{F}}$ is the functional error, defined as the error that would be found if the exact density were used, while $\Delta E_{\mathrm{D}}$ is the (usually much smaller) contribution to the energy error due to the error in the self-consistent density.

\footnotetext{
*esim@yonsei.ac.kr
}

So long as density-driven errors were small compared to the functional errors (as was the case in the halcyon days of B3LYP), they were irrelevant. But in the modern era of vast databases that include weak interactions, stretched bonds, etc., these errors are sometimes as big as (or larger than) the functional errors. ${ }^{17,20}$ However, the common practice of direct comparison with accurate energies conflates both errors and cannot distinguish the two. Recent advances in machine learning of density functionals target the density as well as the energy, and likely succeed because both errors are simultaneously minimized. ${ }^{22}$

The cure for this difficulty is simple: where relevant, empirical schemes should be trained on purely functional errors, i.e., the functional error of a parameterized approximation to the energy should be optimized against accurate energy databases, rather than the self-consistent error. For calculations that are not density-sensitive, the differences are so small as to make this irrelevant. But for those that are, this procedure isolates the self-consistency error and so avoids the corruption of the optimization process, allowing density-sensitive cases to be included even in training.

The current paper highlights the consequences of ignoring this distinction when optimizing parameters in empirical functionals. We first create a totally artificial problem to emphasize the difficulties, especially when one uses a semilocal approximation for the self-consistent density but a more accurate form for the energy. In this case, we show how the exact functional is missed by the standard procedure. Next, we take the D3 correction of Grimme and co-workers, ${ }^{23}$ and show how, if complexes with large density-driven errors are naively included, the results become noticeably worse. On the other hand, the use of DC-DFT allows previous good results to be retained, and the more difficult complexes to be included. We also apply our method to double-hybrids (DHs), producing a combination that competes with similar functionals, but 
still works when the density sensitivity is large. Finally, we find that empirical range-separated hybrid functionals suffer less from density-driven errors than their conventional global counterparts.

For the purposes of this paper, we write a 4-parameter double-hybrid functional $(D H 4 p)$ as:

$$
\begin{aligned}
E_{\mathrm{XC}}^{\mathrm{DH} 4 \mathrm{p}} & =E_{\mathrm{X}}^{\mathrm{Slater}}+\alpha\left(E_{\mathrm{X}}^{\mathrm{HF}}-E_{\mathrm{X}}^{\text {Slater }}\right)+\beta\left(\tilde{E}_{\mathrm{X}}^{\mathrm{GGA}}-E_{\mathrm{X}}^{\text {Slater }}\right) \\
& +\gamma \tilde{E}_{\mathrm{C}}^{\mathrm{GGA}}+\delta E_{\mathrm{C}}^{\text {ab-initio }}
\end{aligned}
$$

where $E_{X}^{\text {Slater }}$ is the local density approximation for exchange, $E_{\mathrm{X}}^{H F}$ is the HF exchange, $\tilde{E}_{\mathrm{X}}^{G G A}$ and $\tilde{E}_{\mathrm{C}}^{G G A}$ denote the approximate GGA exchange and correlation energy, respectively, and $E_{\mathrm{C}}^{a b-i n i t i o}$ is the correlation energy from an $a b$-initio calculation such as MP2. The standard procedure then is to run self-consistent calculations of Eq. 2 without the $a b-$ initio correlation, but evaluate energies with the full $\mathrm{DH}$ expression on the orbitals. ${ }^{24-26}$ The parameters are then chosen to minimize errors for specific molecular datasets. As we show, this assumes that density-driven differences between this and doing the entire procedure self-consistently are negligible.

Often, highly-accurate densities required in Eq. 1 are too expensive to calculate. A practical measure of density sensitivity is given by: ${ }^{19-21}$

$$
\tilde{S}=\left|\tilde{E}\left[n_{\mathrm{LDA}}\right]-\tilde{E}\left[n_{\mathrm{HF}}\right]\right|,
$$

where tilde indicates a given functional approximation. Given the HF tendency to overlocalize, and the LDA tendency to delocalize, and that both are non-empirical, $\tilde{S}$ is a practical guide to the density sensitivity of a given reaction and approximate functional. For small molecules, $\tilde{S}>2 \mathrm{kcal} / \mathrm{mol}$ implies density sensitivity and suggests DC-DFT will improve a functional's performance ${ }^{19}$. In such cases, usually the HF density is sufficient to produce improved energies (HF-DFT).

Illustration: Missing the exact solution for one electronIn this section, we illustrate the dangers of ignoring the distinction between density-driven and functional errors in a simple, toy model: A simplified hybrid applied to the elementary case of $\mathrm{H}_{2}^{+}$as a function of bond length, which is a paradigm of selfinteraction error, or more generally, delocalization error. ${ }^{27,28}$ Standard semilocal approximations yield long-recognized catastrophic errors as the bond is stretched, missing entirely the dissociation limit (see Fig 1 ). ${ }^{28}$ A HF calculation trivially gets this exactly right, since it is exact for (fully spin-polarized) one-electron systems.

Fig. 1(a) shows the exact binding curve (black) easily found by HF, and two other curves of the PBEX evaluated either selfconsistently (blue) or on the HF density (green). The largely irrelevant difference between blue and green curves show that this is a true functional error, not a density-driven one. Even on the exact density, PBE $X$ fails very badly as the bond is stretched. However, the difference in the two curves becomes greater than $2 \mathrm{kcal} / \mathrm{mol}$ at about $1.5 \AA$, showing a density
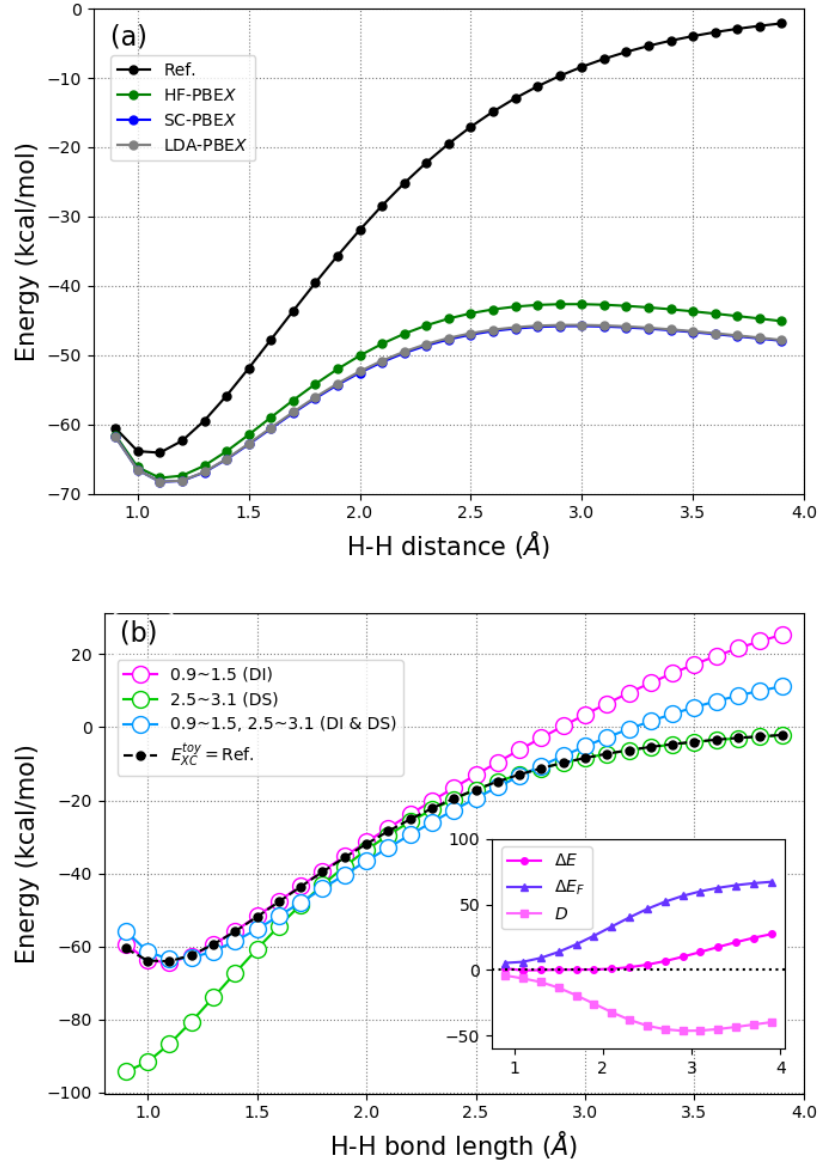

Figure 1: Potential energy surface (PES) of $\mathrm{H}_{2}^{+}$from: (a) exactly (black), self-consistent PBEX (blue) and PBEX on the exact (HF) density (green) and on the LDA density (grey); (b) the toy functional of Eq. 2 with $\gamma=\delta=0$ and no HF in the self-consistent density, with the $a$ and $b$ parameters optimized in different regions: (magenta) the density-insensitive (DI) region $(0.9 \AA-1.5 \AA)$, (green) the density-sensitive $(D S)$ region (2.5A$3.1 \AA)$, (blue) combination of both $D S$ and $D I$ regions. The inset shows $\Delta E$ decomposition for the toy functional trained on the DI region. See also Fig. S1, S2, and Table S2.

sensitivity (the curve with LDA density is indistinguishable from the self-consistent curve) in this problem. (Standard HF-DFT produces accurate curves for heteronuclear diatomics, not homonuclear ones. ${ }^{16,17}$ )

Now, to mimic the standard DH procedure, we perform self-consistent calculations without the HF contribution (since it yields the exact answer in this case), but evaluate the energy with it included. We apply the $\mathrm{DH}$ philosophy to our $\mathrm{H}_{2}^{+}$ molecule, using different separations to generate datasets. Because this is a one-electron system, we simplify the general $\mathrm{DH}$ form to just exchange, setting $\gamma=\delta=0$ in Eq. 2, and use the PBE exchange ${ }^{29}$ as a GGA. Fig. 1(b) shows the results of training in the density-sensitive (stretched, DS) and densityinsensitive (near equilibrium, DI) region of the binding curve. In each case, the optimal parameterization yields accurate 
energies on the training data, but fails badly outside the training range. Even a combination of both equilibrium and stretched data does not help much.

How can this be happening? Obviously, if we set $\alpha=1$ and $\beta=0$ in Eq. 2, we get HF, and so produce the exact answer. But, because the self-consistent calculation uses only a GGA form, which has an unbalanced self-interaction error as the bond is stretched, the exact result is never found. To quantify, we define

$$
D\left[n^{\prime}\right]=\tilde{E}\left[n^{\prime}\right]-\tilde{E}[n],
$$

generalizing ${ }^{30} \Delta E_{\mathrm{D}}$ to arbitrary densities $\left(D[\tilde{n}]=\Delta E_{\mathrm{D}}\right.$, and $D[n]=0)$. We decompose the error for the functional trained near equilibrium, showing $\Delta E_{\mathrm{F}}$ and $D$ in the inset of Fig. 1(b). The optimal parameters (which are nonsensical, see Table S2 of the supporting information) keep the total error to a minimum in the training region where $\Delta E_{\mathrm{F}}$ and $D$ cancel each other by being about equal and opposite. Outside the training region of our $\mathrm{H}_{2}^{+}$curve, this artificial cancellation of errors fails badly. Obviously, we trivially solve this toy problem if we always train on the HF (exact, in this case) density instead of the self-consistent GGA density.

\begin{tabular}{lcccc}
\hline & \multicolumn{2}{c}{$[\mathrm{SC}]$} & \multicolumn{2}{c}{$[\mathrm{HF}]$} \\
opt. dataset & DI & DS & DI & DS \\
\hline without opt. & 1.53 & 2.90 & 1.89 & 4.95 \\
D3 $_{\text {orig }}$ & 0.43 & 6.74 & 0.42 & 1.20 \\
12DB & 0.48 & 5.66 & 0.31 & 0.98 \\
DS-12DB & 1.47 & 2.96 & 0.38 & 0.87 \\
DI-12DB & 0.42 & 6.53 & 0.31 & 1.01 \\
\hline \hline
\end{tabular}

Table 1: Mean absolute errors ( $\mathrm{kcal} / \mathrm{mol})$ of PBE and modifications on density-insensitive (DI) and density-sensitive (DS) test cases (columns) versus optimization on various databases (rows), with self-consistent $(S C)$ densities on left and HF densities on right. $D 3_{\text {orig }}$ denotes the original Grimme dataset, $12 D B$ is our large (320 values) mixed dataset, DI-12DB are its 274 DI cases, and $D S-12 D B$ its $46 D S$ cases.

DFT-D3 for weak interactions- The D3 empirical correction of Grimme and co-workers has become a standard technique for improving the accuracy of DFT approximations when applied to noncovalent interactions. ${ }^{23,31}$ While most such calculations are density insensitive, DFT calculations of specific types of noncovalent interactions, such as halogen bonds, are plagued by density errors, which can be larger than the D3 correction itself. ${ }^{20}$

HF-DFT, as a simple form of DC-DFT, fixes this problem by replacing the SC density with the HF density, on which semilocal functionals yield more accurate energies in such cases. ${ }^{14,19,20,30}$ It was recently shown that the use of the HF density in place of the exact density introduces much smaller errors than the improvements made by HF-DFT. ${ }^{21}$ (As an aside, this does not imply that the pointwise accuracy of the underlying HF density is better than that of SC-DFT densities. ${ }^{19}$ )

The example of Ref. 20 was an extreme case. Here we study the effects of density sensitivity on SC-DFT-D3 calculations of weak interactions when they are more subtle. We use 12 datasets ( 7 from the original D3 parameterization ${ }^{23}$ ) of noncovalent interactions ( 320 data points in total, see Table S1 of the supporting information). ${ }^{32}$ The data points are classified as DS or DI based on their PBE sensitivity, $S^{\mathrm{PBE}}$ (see Eq. 3 and Fig. S3). Only 46 are DS, and these are mostly from B30 ${ }^{33}$ and WATER $27^{32}$, with only one such data point present in the dataset used for the training of the original D3 parameters.

In Table 1, we demonstrate the importance of accounting for the density sensitivity when optimizing parameters for D3 corrections. The first two numbers in the 2 nd column show the dramatic reduction in error in the PBE functional when the original D3 correction is made, on the density-insensitive cases. The next entry shows that when we optimize over our much expanded database, the errors for DI cases are only slightly worse. But if we optimize specifically over our DS cases (4th entry), this greatly worsens results on our DI test cases.

Moving over one column, we find results when tested on the DS cases. Now the original D3 parameterization yields a large (greater than $6 \mathrm{kcal} / \mathrm{mol}$ ) error, demonstrating that density-sensitivity creates large errors. Even when optimized for DS cases, the error remains about $3 \mathrm{kcal} / \mathrm{mol}$.

In the next column, we report the DI test results, but using HF densities instead of SC densities. In all cases of interest, the errors are slightly reduced once D3 with any of the parameters is turned on. The errors fall by more than a factor of 6 if the D3 is trained on the DI cases. Furthermore, the differences between the optimal D3 parameters for DS and DI cases are much smaller when HF densities are used. Fig. 2 shows the variation of the error with parameters. Fig. 2(a) shows the usual case (SC densities on DI cases). Fig. 2(b) is SC densities on DS cases, showing a totally different landscape. A green circle lying at the minimum of the case (a) is denoted in all three panels. Fig. 2(c) is HF densities on DS cases, showing about the same landscape as (a).

Finally, the fourth column of Table 1 shows results on the DS cases using HF densities. While overall, these are much less accurate than the DI cases (by about a factor of 3), they are much better than those of column 2, which uses SC densities

From these findings we can also see the effects of including DS cases in the training set. Their naive inclusion without the density correction via HF-DFT gives some improvements for DS cases at the cost of deteriorated accuracy for DI cases resulting from the abrupt changes in the optimal parameters. On the other hand, after the density correction is applied, the inclusion of DS cases in the training set improves their accuracy without the side effects for DI cases (Table 1) and without abrupt changes in the parameter landscape (Fig. 2).

Most of the DS noncovalent complexes used in the training set in Table 1 belong to the B30 and WATER27 datasets. In Fig. 3, we compare errors of SC-PBE and HF-PBE, with and 

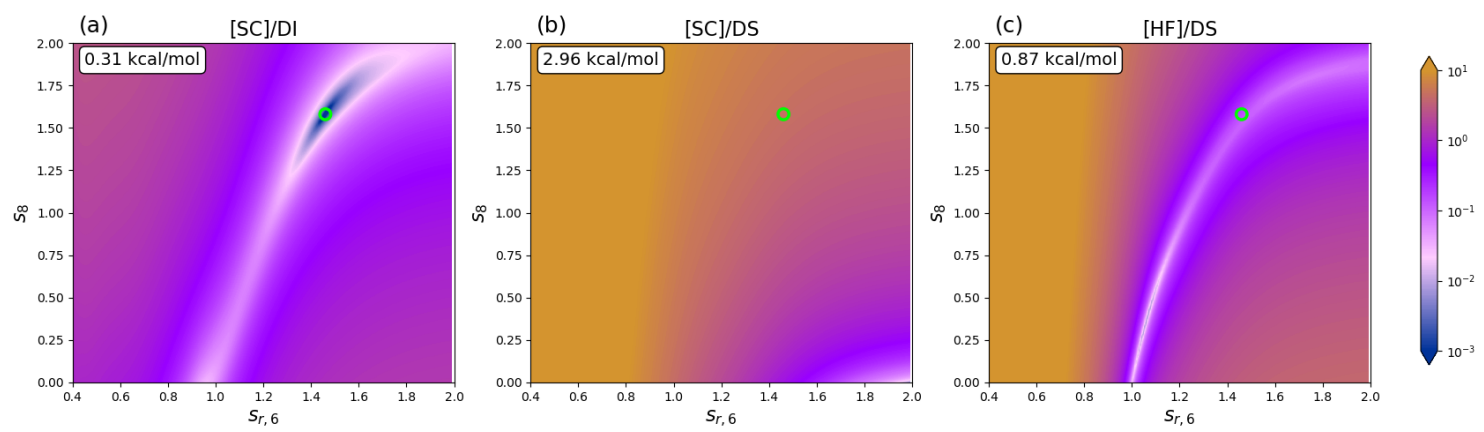

Figure 2: Mean absolute error (MAE) of PBE-D3 as a function of dispersion parameters ${ }^{23}$, for various densities and test sets: (a) self-consistent (SC) density on density-insensitive (DI) cases, (b) SC density for density-sensitive (DS) cases, and (c) HF density for $D S$ cases. Contours are shifted by the minimum value (upper left corner) for clarity. The green circle is at the position of the global minimum of the panel (a).

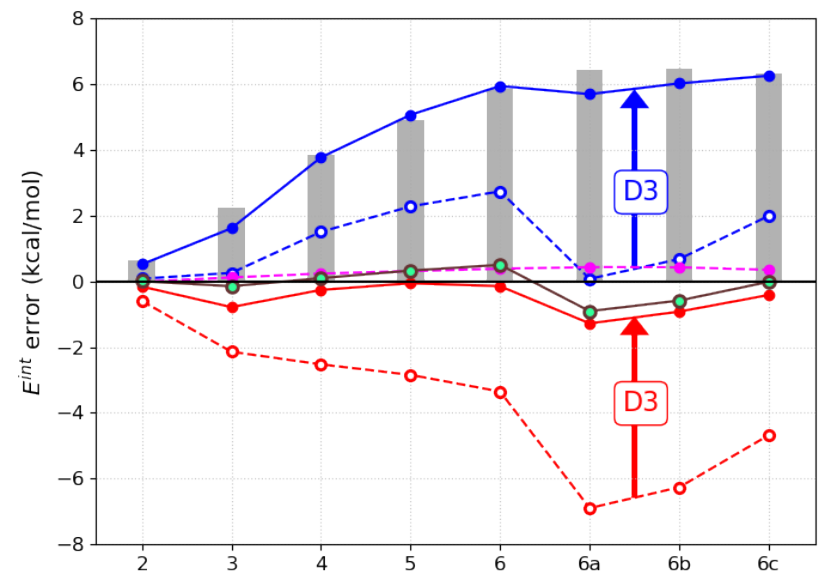

Figure 3: $P B E$ binding energy error for small water clusters, $E^{\mathrm{int}}=n E_{\mathrm{H}_{2} \mathrm{O}}-E_{\left(\mathrm{H}_{2} \mathrm{O}\right)_{\mathrm{n}}}(n=2 \sim 6)$, in WATER27 dataset. Blue denotes self-consistent (PBE), while red is for the HF density (HF-PBE); dashed is without dispersion correction, while solid denotes with D3 (revised is similar to original). The gray bar shows the density-sensitivity of Eq. 3. For comparison, we also show $\omega$ B97M-V (magenta) and BL1p (green, defined later in text) results.

without the (revised)D3 correction, for binding energies of small water clusters of the WATER27 dataset. The standard DFT calculations of these binding energies are highly DS, as shown by the large values for $S^{\mathrm{PBE}}$ shown in Fig. 3.

We see that HF-DFT corrections are larger than D3 here, and that $\mathrm{D} 3$ on self-consistent densities actually corrects in the wrong direction. HF-DFT-D3 reduces errors for the largest clusters from about $6 \mathrm{kcal} / \mathrm{mol}$ to less than $1 \mathrm{kcal} / \mathrm{mol}$, and thus delivers the performance comparable to $\omega B 97 \mathrm{M}-\mathrm{V}^{34}$, which includes nonlocal correlation ${ }^{35}$, and BL1p (a DH that will be introduced later).

Double-hybrids-The energy functional of widely popular $\mathrm{DHs}$ (e.g. Eq. 2) is typically evaluated on the hybrid density and orbitals found in a self-consistent calculation that neglects

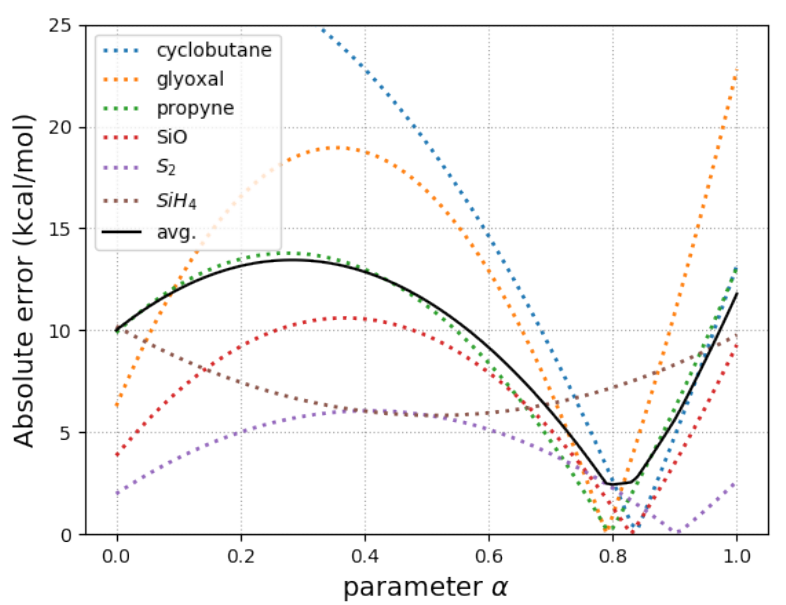

Figure 4: Absolute errors for the AE6 dataset of BL1p as a function of $\alpha$ (see Eq. 5 ) for individual molecules (dashed lines). In the black solid curve, where the averaged errors are shown, the minimum is achieved at $\alpha=0.82$.

the $E_{C}^{a b-i n i t i o}$ term. ${ }^{24,25}$ In contrast, we find that HF-DHs obtained by applying a DH energy expression to the HF density and orbitals yield an overall accuracy competitive with their standard counterparts, but remain accurate for cases where the standard DHs fail due to density sensitivity. We test the HF-DH idea with only one empirical parameter: ${ }^{25}$

$$
\begin{aligned}
E_{\mathrm{XC}}^{\mathrm{DH} 1 \mathrm{p}} & =E_{\mathrm{XC}}^{\mathrm{GGA}}+\alpha\left(E_{\mathrm{X}}^{\mathrm{HF}}-E_{\mathrm{X}}^{\mathrm{GGA}}\right) \\
& +\alpha^{2}\left(E_{\mathrm{C}}^{\mathrm{ab}-\text { initio }}-E_{\mathrm{C}}^{\mathrm{GGA}}\right)
\end{aligned}
$$

as suggested by Sharkas et al. based on adiabatic connection arguments. ${ }^{25}$ (This is Eq. 2 where $\beta=1-\alpha$ and $\delta=1-\gamma=$ $\alpha^{2}$.) To construct a HF-DHs based on Eq. 5, we use here a combination of: B88 exchange ${ }^{1}$, semilocal LYP correlation; ${ }^{2}$ and MP2 correlation for $E_{\mathrm{C}}^{\text {ab-initio } 36}$. We call this functional BL1p. Also, see Fig. S4 to compare 1DH-BLYP (BL1p[SC]) of Ref. 25 and BL1p[HF]. Here we do not aim at reaching 


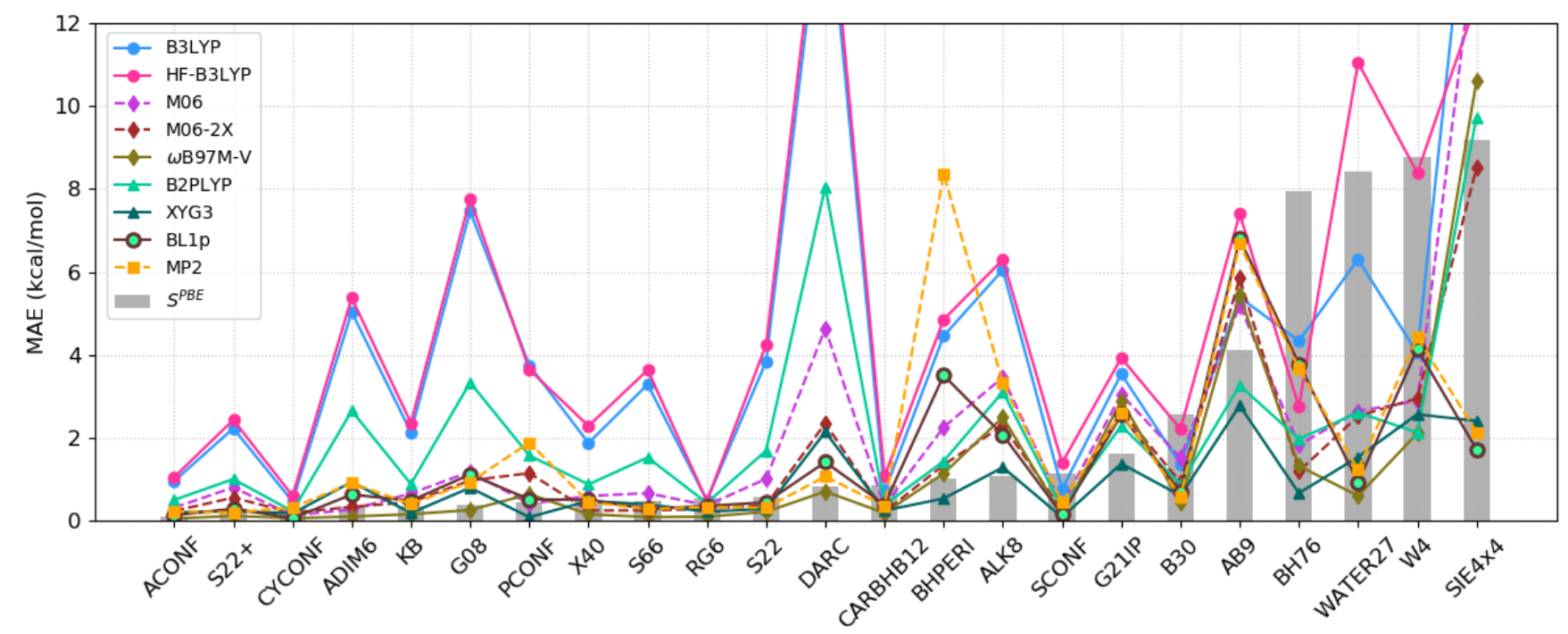

Figure 5: MAEs for several methods on many databases: BL1p, other double-hybrids (B2PLYP and XYG3), hybrids (B3LYP,M06, M06-2X), range-separated meta-GGA hybrid ( $\omega$ B97M-V), and MP2.

the accuracy limit of the HF-DH approach. This is already prohibited by a functional form of Eq. 5, which contains only one empirical parameter. Our goal is to show that this approach delivers an overall performance comparable to the standard DHs while not being plagued by large density-driven errors. Thus, we perform the optimization of $\alpha$ of Eq. 5 in an oldfashioned way, by training BL1p[HF] on the AE6 dataset, containing atomization energies of 6 molecules. ${ }^{37}$ The results of the training are shown in Fig. 4. At $\alpha=0$, our BL1p reduces to HF-BLYP, whereas at $\alpha=1$, it reduces to MP2. The optimal BL1p that minimizes MAE for AE6 has $\alpha=0.82$, which varies little between molecules, except for $\mathrm{SiH}_{4}$ whose minimum is much shallower. Also, the MAE of optimal BL1p is about $7.5 \mathrm{kcal} / \mathrm{mol}$ smaller than the $\alpha=0$ case (HF-BLYP) and about $9 \mathrm{kcal} / \mathrm{mol}$ than the $\alpha=1$ case (MP2).

In Fig. 5, we compare the performance of BL1P with the standard DHs (B2PLYP ${ }^{24}$ and $X Y G 3^{38}$ ), hybrids (B3LYP, M06, M06-2X), and also with the range-separated functional $\left(\omega \mathrm{B} 97 \mathrm{M}-\mathrm{V}^{34}\right)$, which we detail in the supporting information. This figure shows that the one-parameter BL1p, trained only 6 atomization energies, yields an accuracy that is competitive with the standard DHs for all databases, and works for noncovalent interactions, without using Grimme's empirical correction. Usually, we recommend against using the HF density when it suffers from spin-contamination. ${ }^{14}$ Nevertheless, for all data in this section, we include the spin-contaminated cases for fair comparison. Performance without spin-contaminated cases is shown in the supporting information (see Table S3).

Returning to our starting point, stretched $\mathrm{NaCl}$ is a prototypical case where self-consistent hybrids and GGAs are contaminated by large density errors. ${ }^{16}$ These errors are typical of semilocal functionals for dissociating heterodimers. ${ }^{39,40}$ HF densities fix this problem, and HF-DFT is able to disso-

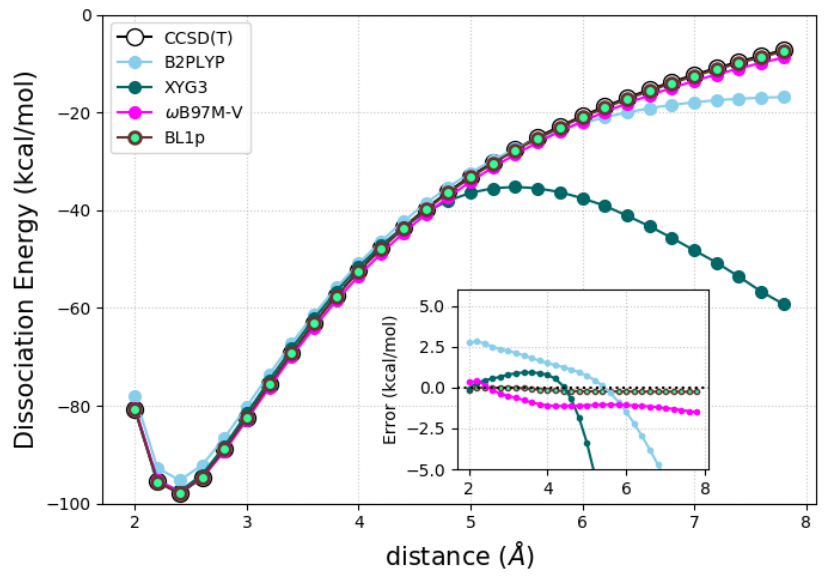

Figure 6: Dissociation curves of $\mathrm{NaCl}$ obtained from various approaches. For stretched bond lengths, standard double-hybrid functionals fail due to the density-driven errors (see Ref. 16).

ciate heterodimers correctly. ${ }^{16}$ From Fig. 6 , in contrast to a standard DHs (B2PLYP and XYG3 shown here) that fail at large bond lengths, our BL1p, as a representative of HF-DH, dissociates $\mathrm{NaCl}$ correctly (See also Fig S6).

Another case where BL1p outperforms other methods is the SIE4 4 dataset, containing four positively charged dimers at four different separations, where standard DFT methods have large self-interaction error. ${ }^{32}$ Fig. 7 shows the dissociation curve of $\mathrm{He}_{2}^{+}$, as a representative of this dataset. First, the errors of the standard DFT methods for $\mathrm{He}_{2}^{+}$are almost entirely functional errors (see Fig. S5), because they differ little between accurate and self-consistent densities. The accurate densities are obtained by Kohn-Sham inversion from CCSD densities. ${ }^{21}$ In this way, the source of error of the standard DFT for $\mathrm{He}_{2}^{+}$is 


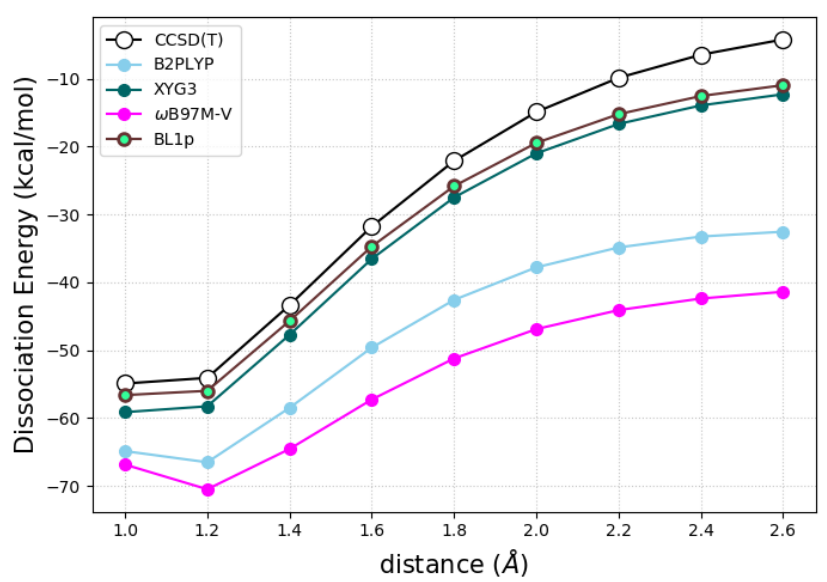

Figure 7: Dissociation curve of $\mathrm{He}_{2}^{+}$obtained from various functionals. See also Fig. S5, showing that the errors of standard functionals for $\mathrm{He}_{2}^{+}$are mostly functional errors, since self-consistent results are almost identical to those when the functionals are applied to accurate densities (obtained from the Kohn-Sham inversion scheme from the CCSD wavefunction ${ }^{21}$ ).

very different from that of stretched $\mathrm{NaCl}$. Fig. 7 shows that, even though these are not density-driven errors, the error of BL1p for $\mathrm{He}_{2}^{+}$is much smaller than that of other approaches.

Range-separated vs. conventional hybrids-We have shown a number of examples where large density-driven errors of conventional (global) hybrid functionals are substantially reduced when they are evaluated on the HF instead of SC densities. Range-separated hybrids (RSHs) often use $100 \%$ of the $\mathrm{HF}$ exchange in the long-range $(\mathrm{Ir})^{34,41,42}$, and so should suffer less from density-driven errors. ${ }^{43}$. To test this, we use $\omega \mathrm{B} 97 \mathrm{M}-\mathrm{V}$ as a representative of $\mathrm{RSHs}^{34}$, given its remarkable performance for many of the databases in Fig. 5. We will compare $\omega \mathrm{B} 97 \mathrm{M}-\mathrm{V}$ with $\mathrm{B} 97 \mathrm{M}-\mathrm{V}$, its conventional analog. ${ }^{44}$. The density-driven errors of $\omega \mathrm{B} 97 \mathrm{M}-\mathrm{V}$ and $\mathrm{B} 97 \mathrm{M}-\mathrm{V}$ are shown in Fig. 8 for our two standard cases, with PBE and B3LYP also shown for comparison. For $\mathrm{H}_{2}^{+}$, the $\mathrm{HF}$ density is exact, while for $\mathrm{NaCl}$, we invert the accurate Kohn-Sham density from CCSD. ${ }^{21}$ In each case, the density-driven error of $\omega \mathrm{B} 97 \mathrm{M}-\mathrm{V}$ is much smaller than that of the other functionals. It does not vanish, because of the semilocal part of the functional. We see similar behavior for larger systems where the error of conventional hybrid functionals is contaminated by the densities, and is much smaller in $\omega B 97 M-V$. Sensitivity plots are used as a diagnostic tool for density-driven errors, and in Fig. 9 we show that the sensitivity of $\omega B 97 \mathrm{M}-\mathrm{V}$ for the WATER27 complexes is a fraction of that of B3LYP and B97M-V.

Concluding remarks- We have shown the dangers of ignoring density errors in the construction of empirical approximations. In our simple $\mathrm{H}_{2}^{+}$example, a parameterized semilocal functional trained on a limited region of the $\mathrm{H}_{2}^{+}$binding curve fails in all other regions. Even high accuracy in the training region results from an enforced error cancellation between the
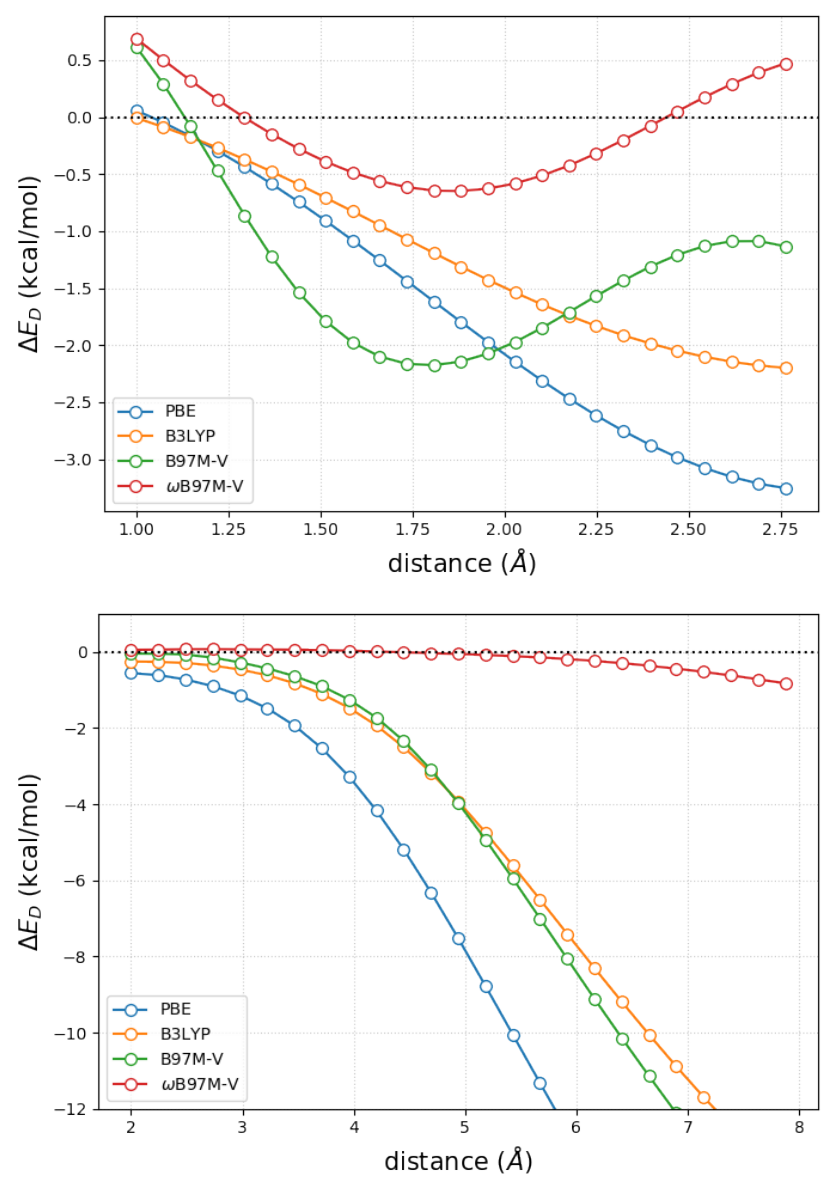

Figure 8: Density-driven errors (see Eq. 1) of selected functionals along the dissociation curves of:e $H_{2}^{+}$(top panel) and $\mathrm{NaCl}$ (bottom panel). For $\mathrm{H}_{2}^{+}$, the (exact) $\mathrm{HF}$ density is used to extract the density-driven errors. For $\mathrm{NaCl}$, we use CCSD as a reference in tandem with the Kohn-Sham inversion scheme described in Ref. 21 to obtain the 'exact' density and orbitals needed to isolate density-driven errors.

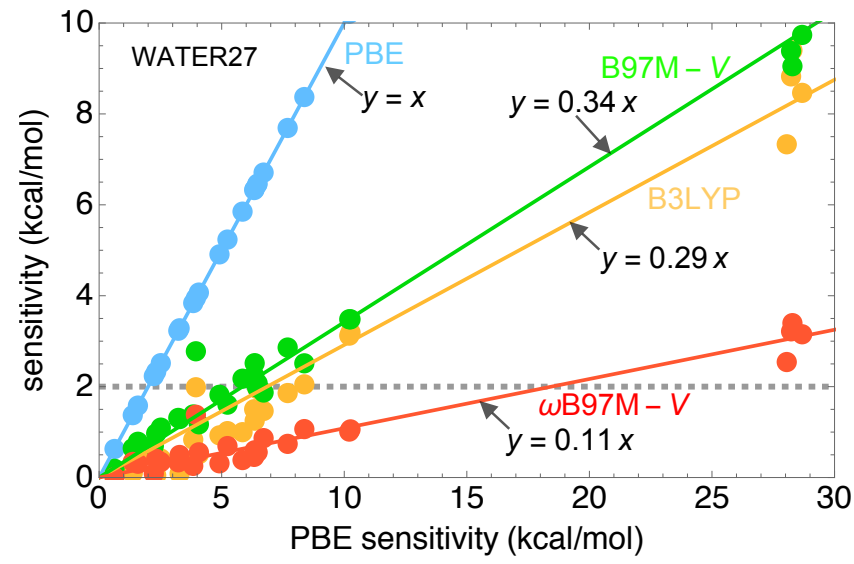

Figure 9: The sensitivity (see Eq. 3) of selected functionals vs. PBE sensitivities for binding energies of the WATER27 clusters.

density and functional error (Eq. 1), which fails outside this 
region. We found that the standard DFT with empirical D3 corrections breaks down in density-sensitive calculations of noncovalent systems, but is fixed by using the HF density.

We also found that resilience to density-driven errors could be achieved with simple 1-parameter double-hybrids, once they are trained and applied to HF densities. As always, our use of HF densities does not imply that they are point-wise more accurate than self-consistent densities, but simply that they yield more accurate energetics when a reaction is density sensitive. Our BL1p is trained only on atomization energies of only 6 molecules, but its accuracy is comparable to the standard doubled hybrids tested here. Moreover, $\omega B 97 \mathrm{M}-\mathrm{V}$ outperforms BL1p for most of the datasets considered in Fig 5, except for the SIE4x4 dataset, where BL1p does much better. BL1p would also be beaten by $\omega \mathrm{B} 97(2)$, a very recent highly accurate $\mathrm{DH}$ designed to improve over $\omega \mathrm{B} 97 \mathrm{M}-\mathrm{V} .{ }^{45}$ Given its excellent performance ${ }^{45,46}$, we expect it to beat BL1p on most of the datasets, but not SIE $4 \times 4$.

Our goal here is not the introduction of a new empirical XC functional, but to illustrate contamination due to density errors in fitting procedures and to show how minimizing the functional error can improve the performance of empirical functionals. Thus, our primitively optimized BL1p does not reach the accuracy limit of the HF-DH class of functionals. Technical advances in optimization and larger parameter spaces could further improve its accuracy. Furthermore, to improve HF$\mathrm{DHs}$, one may also use the new insights into functionals that explicitly depend on the HF density obtained from the adiabatic connection that has the MP2 theory as its weak-interaction expansion. ${ }^{47,48}$ Finally, we have found that using $100 \%$ of HF exchange in range-separated hybrids means they suffer much less from density-driven errors than their conventional counterparts.

In summary, DFT energy errors can be separated into functional and density-driven using DC-DFT. To avoid inaccuracies, empirical functionals can be trained on functional errors only, where practical. In cases of large density sensitivity, HF densities (unless flawed by, e.g., spin-contamination) are typically more useful than self-consistent semilocal densities. With $100 \%$ exchange at large distances, range separated functionals are relatively density insensitive, and suffer much less from these issues.

\section{Computational Details}

All HF, DFT, HF-DFT, and MP2 calculations have been performed with the TURBOMOLE v7.0.2. ${ }^{49}$ and PYSCF v1.7.2. ${ }^{50}$ The following functionals have been used in DFT and HF-DFT calculations: LDA (SVWN ${ }^{51,52}$ ), GGA (PBE $\left.{ }^{29}, \mathrm{BLYP}^{1,2}\right)$, mGGA (TPSS ${ }^{53}$ ), hybrids (B3LYP ${ }^{4}, \mathrm{PBE}^{54}$, M06, M06$2 \mathrm{X}^{55}, \mathrm{~B} 97 \mathrm{M}-\mathrm{V}^{44}, \omega \mathrm{B} 97 \mathrm{M}-\mathrm{V}^{34}, \mathrm{~B} 2 \mathrm{PLYP}^{24}$, and $\mathrm{XYG}^{38}$ ). The scripts for performing HF-DFT energy calculations are available. ${ }^{56}$ Unless otherwise stated, the def2-QZVPPD basis set has been used. All geometries and the multiplicities except for the AE6 ${ }^{37}$ have been taken from Ref. 32. Further computational details can be found in the supporting information.

\section{SUPPLEMENTARY INFORMATION}

- Dataset description

- Optimized parameters and mean absolute error for $\mathrm{H}_{2}^{+}$

- Mean absolute error value of Fig. 5

\section{ACKNOWLEDGEMENT}

This work at Yonsei University was supported by the grant from the Korean Research Foundation (NRF-2020R1A2C2007468 and NRF-2020R1A4A1017737). KB acknowledges funding from NSF (CHEM 1856165). SV acknowledges funding from the Rubicon project (019.181EN.026), which is financed by the Netherlands Organisation for Scientific Research (NWO). We thank Professor Martin Head-Gordon and Dr. Narbe Mardirossian for stimulating discussions.

\section{REFERENCES}

[1] Axel D Becke. Density-functional exchange-energy approximation with correct asymptotic behavior. Physical Review A, 38(6):3098-3100, 1988.

[2] Chengteh Lee, Weitao Yang, and Robert G Parr. Development of the colle-salvetti correlation-energy formula into a functional of the electron density. Physical Review B, 37(2):785-789, 1988.

[3] Axel D Becke. Density-functional thermochemistry. iii. the role of exact exchange. The Journal of Chemical Physics, 98(7):5648-5652, 1993.

[4] P. J. Stephens, F. J. Devlin, C. F. Chabalowski, and M. J. Frisch. Ab initio calculation of vibrational absorption and circular dichroism spectra using density functional force fields. The Journal of Physical Chemistry, 98(45):1162311627, 1994.

[5] Haoyu S Yu, Shaohong L Li, and Donald G Truhlar. Perspective: Kohn-sham density functional theory descending a staircase. The Journal of Chemical Physics, 145(13):130901, 2016.

[6] Narbe Mardirossian and Martin Head-Gordon. Thirty years of density functional theory in computational chemistry: an overview and extensive assessment of 200 density functionals. Molecular Physics, 115(19):2315-2372, 2017.

[7] Michael G Medvedev, Ivan S Bushmarinov, Jianwei Sun, John P Perdew, and Konstantin A Lyssenko. Density 
functional theory is straying from the path toward the exact functional. Science, 355(6320):49-52, 2017.

[8] Enrico Clementi and Subhas J Chakravorty. A comparative study of density functional models to estimate molecular atomization energies. The Journal of Chemical Physics, 93(4):2591-2602, 1990.

[9] Nevin Oliphant and Rodney J Bartlett. A systematic comparison of molecular properties obtained using hartreefock, a hybrid hartree-fock density-functional-theory, and coupled-cluster methods. The Journal of Chemical Physics, 100(9):6550-6561, 1994.

[10] Benjamin G Janesko and Gustavo E Scuseria. Hartreefock orbitals significantly improve the reaction barrier heights predicted by semilocal density functionals. The Journal of Chemical Physics, 128(24):244112, 2008.

[11] David RB Brittain, Ching Yeh Lin, Andrew TB Gilbert, Ekaterina I Izgorodina, Peter MW Gill, and Michelle L Coote. The role of exchange in systematic dft errors for some organic reactions. Physical Chemistry Chemical Physics, 11(8):1138-1142, 2009.

[12] Min-Cheol Kim, Eunji Sim, and Kieron Burke. Communication: Avoiding unbound anions in density functional calculations. The Journal of Chemical Physics, 134(17):171103, 2011.

[13] Prakash Verma, Ajith Perera, and Rodney J. Bartlett. Increasing the applicability of dft i: Non-variational correlation corrections from hartree-fock dft for predicting transition states. Chemical Physics Letters, 524:10 - 15, 2012.

[14] Min-Cheol Kim, Eunji Sim, and Kieron Burke. Understanding and reducing errors in density functional calculations. Physical Review Letters, 111(7):073003, 2013.

[15] Min-Cheol Kim, Eunji Sim, and Kieron Burke. Ions in solution: Density corrected density functional theory (dcdft). The Journal of Chemical Physics, 140(18):18A528, 2014.

[16] Min-Cheol Kim, Hansol Park, Suyeon Son, Eunji Sim, and Kieron Burke. Improved dft potential energy surfaces via improved densities. The Journal of Physical Chemistry Letters, 6(19):3802-3807, 2015.

[17] Adam Wasserman, Jonathan Nafziger, Kaili Jiang, MinCheol Kim, Eunji Sim, and Kieron Burke. The importance of being self-consistent. Annual Review of Physical Chemistry, 68(1):555-581, 2017.

[18] Suhwan Song, Min-Cheol Kim, Eunji Sim, Anouar Benali, Olle Heinonen, and Kieron Burke. Benchmarks and reliable dft results for spin gaps of small ligand fe (ii) complexes. Journal of Chemical Theory and Computation, 14(5):2304-2311, 2018.

[19] Eunji Sim, Suhwan Song, and Kieron Burke. Quantifying density errors in dft. The Journal of Physical Chemistry Letters, 9(22):6385-6392, 2018.

[20] Yeil Kim, Suhwan Song, Eunji Sim, and Kieron Burke. Halogen and chalcogen binding dominated by densitydriven errors. The Journal of Physical Chemistry Letters, 10(2):295-301, 2018.

[21] Seungsoo Nam, Suhwan Song, Eunji Sim, and Kieron Burke. Measuring density-driven errors using Kohn-Sham inversion. Journal of Chemical Theory and Computation, 16(8):5014-5023, 2020.

[22] Ryo Nagai, Ryosuke Akashi, and Osamu Sugino. Completing density functional theory by machine learning hidden messages from molecules. npj Computational Materials, 6(1):1-8, 2020.

[23] Stefan Grimme, Jens Antony, Stephan Ehrlich, and Helge Krieg. A consistent and accurate ab initio parametrization of density functional dispersion correction ( $\mathrm{dft}$-d) for the 94 elements h-pu. The Journal of Chemical Physics, 132(15):154104, 2010.

[24] Stefan Grimme. Semiempirical hybrid density functional with perturbative second-order correlation. The Journal of Chemical Physics, 124(3):034108, 2006.

[25] Kamal Sharkas, Julien Toulouse, and Andreas Savin. Double-hybrid density-functional theory made rigorous. The Journal of Chemical Physics, 134(6):064113, 2011.

[26] Jan ML Martin and Golokesh Santra. Empirical DoubleHybrid Density Functional Theory: A 'Third Way'in Between WFT and DFT. Israel Journal of Chemistry, 60:1$19,2019$.

[27] Aron J. Cohen, Paula Mori-Sánchez, and Weitao Yang. Insights into current limitations of density functional theory. Science, 321(5890):792-794, 2008.

[28] Aron J Cohen, Paula Mori-Sánchez, and Weitao Yang. Challenges for density functional theory. Chemical Reviews, 112(1):289-320, 2011.

[29] John P Perdew, Kieron Burke, and Matthias Ernzerhof. Generalized gradient approximation made simple. Physical Review Letters, 77(18):3865, 1996.

[30] Stefan Vuckovic, Suhwan Song, John Kozlowski, Eunji Sim, and Kieron Burke. Density functional analysis: The theory of density-corrected dft. Journal of Chemical Theory and Computation, 15(12):6636-6646, 2019. 
[31] Eike Caldeweyher, Sebastian Ehlert, Andreas Hansen, Hagen Neugebauer, Sebastian Spicher, Christoph Bannwarth, and Stefan Grimme. A generally applicable atomic-charge dependent london dispersion correction. The Journal of Chemical Physics, 150(15):154122, 2019.

[32] Lars Goerigk, Andreas Hansen, Christoph Bauer, Stephan Ehrlich, Asim Najibi, and Stefan Grimme. A look at the density functional theory zoo with the advanced gmtkn55 database for general main group thermochemistry, kinetics and noncovalent interactions. Physical Chemistry Chemical Physics, 19(48):32184-32215, 2017.

[33] Antonio Bauza, Ibon Alkorta, Antonio Frontera, and Jose Elguero. On the reliability of pure and hybrid dft methods for the evaluation of halogen, chalcogen, and pnicogen bonds involving anionic and neutral electron donors. Journal of Chemical Theory and Computation, 9(11):5201-5210, 2013.

[34] Narbe Mardirossian and Martin Head-Gordon. wb97mv: A combinatorially optimized, range-separated hybrid, meta-gga density functional with vv10 nonlocal correlation. The Journal of Chemical Physics, 144(21):214110, 2016.

[35] Oleg A. Vydrov and Troy Van Voorhis. Nonlocal van der waals density functional: The simpler the better. The Journal of Chemical Physics, 133(24):244103, December 2010.

[36] Chr. Møller and M. S. Plesset. Note on an approximation treatment for many-electron systems. Physical Review, 46(7):618-622, October 1934.

[37] Benjamin J Lynch and Donald G Truhlar. Small representative benchmarks for thermochemical calculations. The Journal of Physical Chemistry A, 107(42):8996-8999, 2003.

[38] Ying Zhang, Xin Xu, and William A Goddard. Doubly hybrid density functional for accurate descriptions of nonbond interactions, thermochemistry, and thermochemical kinetics. Proceedings of the National Academy of Sciences, 106(13):4963-4968, 2009.

[39] Oleg V Gritsenko and Evert Jan Baerends. Effect of molecular dissociation on the exchange-correlation kohnsham potential. Physical Review A, 54(3):1957, 1996.

[40] Sara Giarrusso, Stefan Vuckovic, and Paola Gori-Giorgi. Response potential in the strong-interaction limit of $\mathrm{dft}$ : Analysis and comparison with the coupling-constant average. Journal of Chemical Theory and Computation, 9(11):3137-3142, 2018.

[41] Oleg A. Vydrov and Gustavo E. Scuseria. Assessment of a long-range corrected hybrid functional. The Journal of Chemical Physics, 125(23):234109, December 2006.
[42] Narbe Mardirossian and Martin Head-Gordon. wb97xv: A 10-parameter, range-separated hybrid, generalized gradient approximation density functional with nonlocal correlation, designed by a survival-of-the-fittest strategy. Physical Chemistry Chemical Physics, 16(21):9904, 2014.

[43] Erin R. Johnson, A. Otero de-la Roza, and Stephen G. Dale. Extreme density-driven delocalization error for a model solvated-electron system. The Journal of Chemical Physics, 139(18):184116, November 2013.

[44] Narbe Mardirossian and Martin Head-Gordon. Mapping the genome of meta-generalized gradient approximation density functionals: The search for b97m-v. The Journal of Chemical Physics, 142(7):074111, February 2015.

[45] Narbe Mardirossian and Martin Head-Gordon. Survival of the most transferable at the top of Jacob's ladder: Defining and testing the $\omega$ B97M (2) double hybrid density functional. The Journal of Chemical Physics, 148(24):241736, 2018.

[46] Golokesh Santra, Nitai Sylvetsky, and Jan M. L. Martin. Minimally empirical double-hybrid functionals trained against the GMTKN55 database: revDSD-PBEP86-d4, revDOD-PBE-d4, and DOD-SCAN-d4. The Journal of Physical Chemistry A, 123(24):5129-5143, May 2019.

[47] Michael Seidl, Sara Giarrusso, Stefan Vuckovic, Eduardo Fabiano, and Paola Gori-Giorgi. Communication: Stronginteraction limit of an adiabatic connection in hartree-fock theory. The Journal of Chemical Physics, 149(24):241101, December 2018.

[48] Timothy J. Daas, Juri Grossi, Stefan Vuckovic, Ziad H. Musslimani, Derk P. Kooi, Michael Seidl, Klaas J. H. Giesbertz, and Paola Gori-Giorgi. Large coupling-strength expansion of the møller-plesset adiabatic connection: From paradigmatic cases to variational expressions for the leading terms, 2020.

[49] TURBOMOLE V7.0 2015, a development of University of Karlsruhe and Forschungszentrum Karlsruhe $\mathrm{GmbH}$, 1989-2007, TURBOMOLE GmbH, since 2007; available from http://www. turbomole.com.

[50] Qiming Sun and Timothy C. Berkelbach and Nick S. Blunt and George H. Booth and Sheng Guo and Zhendong $\mathrm{Li}$ and Junzi Liu and James D. McClain and Elvira R. Sayfutyarova and Sandeep Sharma and Sebastian Wouters and Garnet Kin-Lic Chan. PySCF: the Python-based simulations of chemistry framework, 2017.

[51] Paul AM Dirac. Note on exchange phenomena in the thomas atom. In Mathematical Proceedings of the Cambridge Philosophical Society, volume 26, pages 376-385. Cambridge University Press, 1930. 
[52] Seymour H Vosko, Leslie Wilk, and Marwan Nusair. Accurate spin-dependent electron liquid correlation energies for local spin density calculations: a critical analysis. Canadian Journal of Physics, 58(8):1200-1211, 1980.

[53] Jianmin Tao, John P Perdew, Viktor N Staroverov, and Gustavo E Scuseria. Climbing the density functional ladder: Nonempirical meta-generalized gradient approximation designed for molecules and solids. Physical Review Letters, 91(14):146401, 2003.

[54] Kieron Burke, Matthias Ernzerhof, and John P Perdew. The adiabatic connection method: a non-empirical hybrid. Chemical Physics Letters, 265:115-120, 1997.

[55] Yan Zhao and Donald G Truhlar. The m06 suite of density functionals for main group thermochemistry, thermochemical kinetics, noncovalent interactions, excited states, and transition elements: two new functionals and systematic testing of four m06-class functionals and 12 other functionals. Theoretical Chemistry Accounts, 120(13):215-241, 2008.

[56] http://tccl.yonsei.ac.kr/mediawiki/index. php/DC-DFT. 


\section{Supporting Information for "Density sensitivity of empirical functionals"}

Suhwan Song ${ }^{a}, \mathrm{Stefan} \mathrm{Vuckovic}^{b}$, Eunji Sim ${ }^{a, *}$, And Kieron Burke ${ }^{b}$

${ }^{a}$ Department of Chemistry, Yonsei University, 50 Yonsei-ro Seodaemun-gu, Seoul 03722, Korea

${ }^{b}$ Departments of Chemistry and of Physics, University of California, Irvine, CA 92697, USA

December 2, 2020

\section{List OF TABLES}

S1 Dataset description ............ . 2

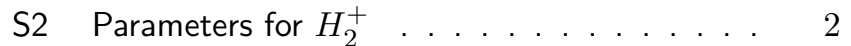

S3 MAE tables of Fig. $5 \ldots \ldots$........ 5

\section{List of Figures}

S1 $\Delta E$ decomposition for $H_{2}^{+}$curve. . . . . . 3

S2 $H_{2}^{+}$PES curve of BL1p . . . . . . . . . 3

S3 Densitiy sensitivity for each dataset . . . . . . 3

S4 BL1p for AE6 dataset . . . . . . . . . . . . . 4

S5 $\mathrm{He}_{2}^{+}$PES curve .............. . . 4

S6 XYG3 for $\mathrm{NaCl}$ PES curve ......... 4

*esim@yonsei.ac.kr 


\section{i. Computational Details}

All HF, DFT, HF-DFT, and MP2 calculations have been performed with the TURBOMOLE v7.0.2.[49] and PYSCF v1.7.2.[50] The following functionals have been used in DFT and HF-DFT calculations: LDA (SVWN[51, 52]), GGA (PBE[29], BLYP[1, 2]), mGGA (TPSS[53]), hybrids (B3LYP[4], PBE0[54], M06, M06-2X[55], B97M-V[44], $\omega B 97 M-V[34]$, B2PLYP[24], and XYG3[38]). The scripts for performing HFDFT energy calculations are available.[57] Unless otherwise stated, the def2-QZVPPD basis set has been used. For accelerating the self-consistent field (SCF) procedure, we adopted the resolution of the identity approximation (RI-J) with def2QZVPPD auxiliary basis set for G08, PCONF, SCONF, and WATER27 dataset. For the KB dataset, we omit the noble gas and the S22 dataset from the original KB65. The energy and one-electron density convergence threshold have been set to $1 \mathrm{e}-8$ and $1 \mathrm{e}-6$ a.u., respectively. Numerical quadrature grids of size 4 have been used (grid size 4 in TURBOMOLE). For VV10 correlation, 99 radial shells with 590 angular grid points per shell are used with the SG1 prune. For all open shell calculation, unrestricted scheme is used. The parameters of Table S2 are first found in the global optimizer shgo and then optimized locally with the Nelder-Mead method in the SCIPY package with the 1e-8 convergence criterion. For revised D3, we scanned $0.00<s_{r, 6}<2.00$ and $0.00<s_{8}<2.00(0.01$ grid spacing) for all 320 datapoints in 12 databases (DB) (marked by an asterisks in table S1) for all XC functionals except $\omega \mathrm{B} 97 \mathrm{M}-\mathrm{V}, \mathrm{XYG} 3$ and HF-DHs. The $\alpha$ value for HF-DHs is optimized with the slsqp method in the SCIPY package. All geometries and the multiplicities except for the AE6 [37] have been taken from Ref. [32].

\begin{tabular}{lrl}
\hline DB & no. points & Description \\
\hline RG6 $^{*}$ & 6 & rare gases \\
ACONF* $^{*}$ & 15 & alkane conformers \\
S22+ $^{*}$ & 66 & non-covalent interaction \\
CYCONF* $^{*}$ & 10 & cysteine conformers \\
ADIM6* $^{*}$ & 6 & alkane dimers \\
$\mathrm{KB}^{\dagger}$ & 27 & non-covalent interactions \\
G08 & 6 & pi-interaction of small carbon complexes \\
PCONF* $^{*} 66^{\dagger}$ & 10 & peptide conformers \\
X40 $^{\dagger}$ & 66 & non-covalent interactions \\
S22 $_{\text {DARC }}$ & 40 & halogen interactions \\
CARBHB12 & 22 & noncovalently bound dimers \\
BHPERI & 14 & Diels-Alder reactions \\
G21IP & 12 & hydrogen-bonded \\
ALK8 & 26 & pericyclic reactions \\
SCONF* & 36 & adiabatic ionization potentials \\
B30 & 8 & alkaline compounds \\
AB9 & 17 & sugar conformers \\
SIE4x4 & 30 & non-covalent interactions for halogen \\
BH76 & 9 & anomalous barrier height \\
W4 & 16 & self-interaction-error \\
WATER27 & 76 & reaction barrier height \\
\hline \hline
\end{tabular}

Table S1: Data sets used in this work. The asterisks indicate the data sets used for optimization of the original D3 parameters by Grimme and co-workes as described in Ref. [23]. For the revised D3, additional 5 datasets (KB, S66, X40, B30, and WATER27) are used as a training set and marked by a dagger.

\begin{tabular}{cccrrr}
\hline$R_{\mathrm{H}-\mathrm{H}}$ & $a$ & $b$ & training & test & all \\
\hline $0.9 \sim 1.5$ & 1.397 & 6.799 & 0.30 & 10.15 & 7.93 \\
$2.5 \sim 3.1$ & 1.237 & -1.532 & 0.02 & 6.64 & 5.14 \\
$0.9 \sim 1.5 \& 2.5 \sim 3.1$ & 1.218 & 4.665 & 2.22 & 6.85 & 4.76 \\
\hline
\end{tabular}

Table S2: Optimized parameters in Fig. 1 and mean absolute error (MAE) in kcal/mol. Note that when the toy model is applied to the $H F$ densities, we get $a=1$ and $b=0$ regardless of the training set, and thereby both the density-driven and functional errors are eliminated. 

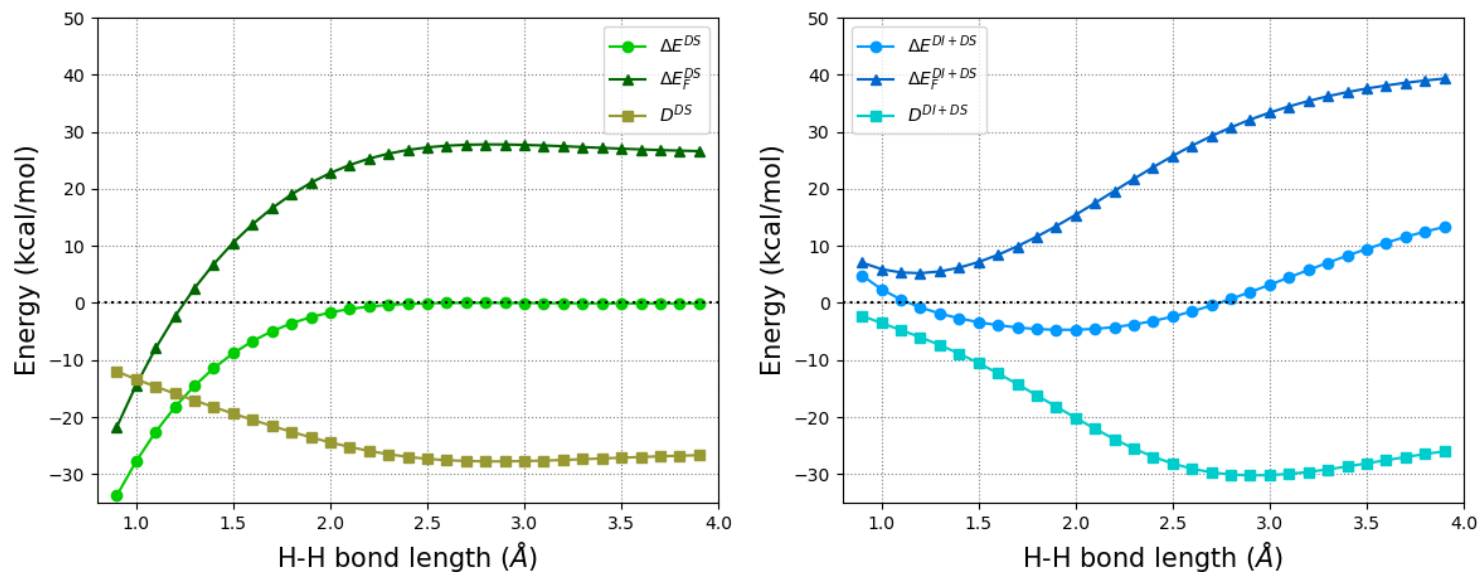

Figure S1: $D$ and $\Delta E_{F}$ error decomposition for $H_{2}^{+}$dissociation curve of the empirical toy functional trained on the DS region (left panel) and on the combination of DS and DI regions (right panel) when applied to $H_{2}^{+}$along the dissociation curve. The same result, but for the functional trained on the DI region is shown in the inset of the bottom panel of of Fig. 1.

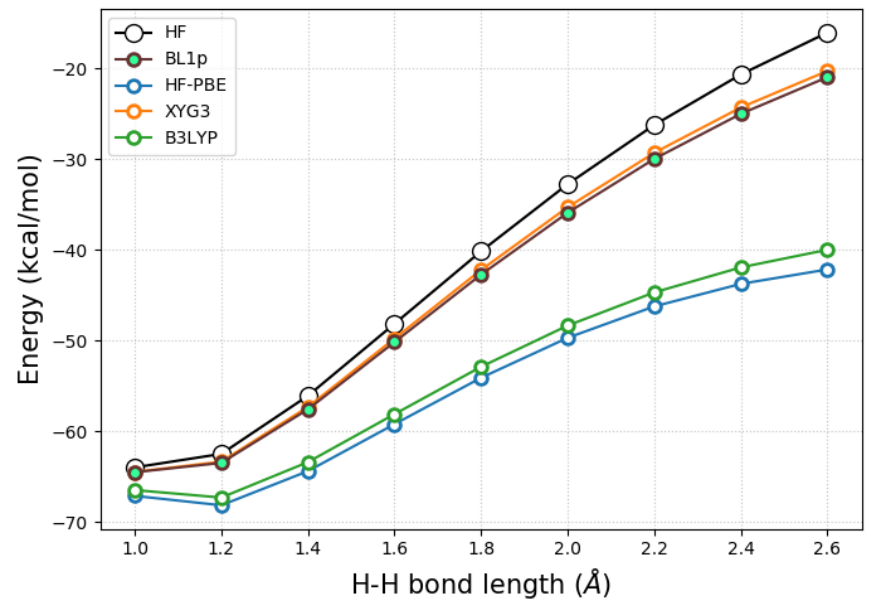

Figure S2: $H_{2}^{+}$potential energy surface for the various methods.

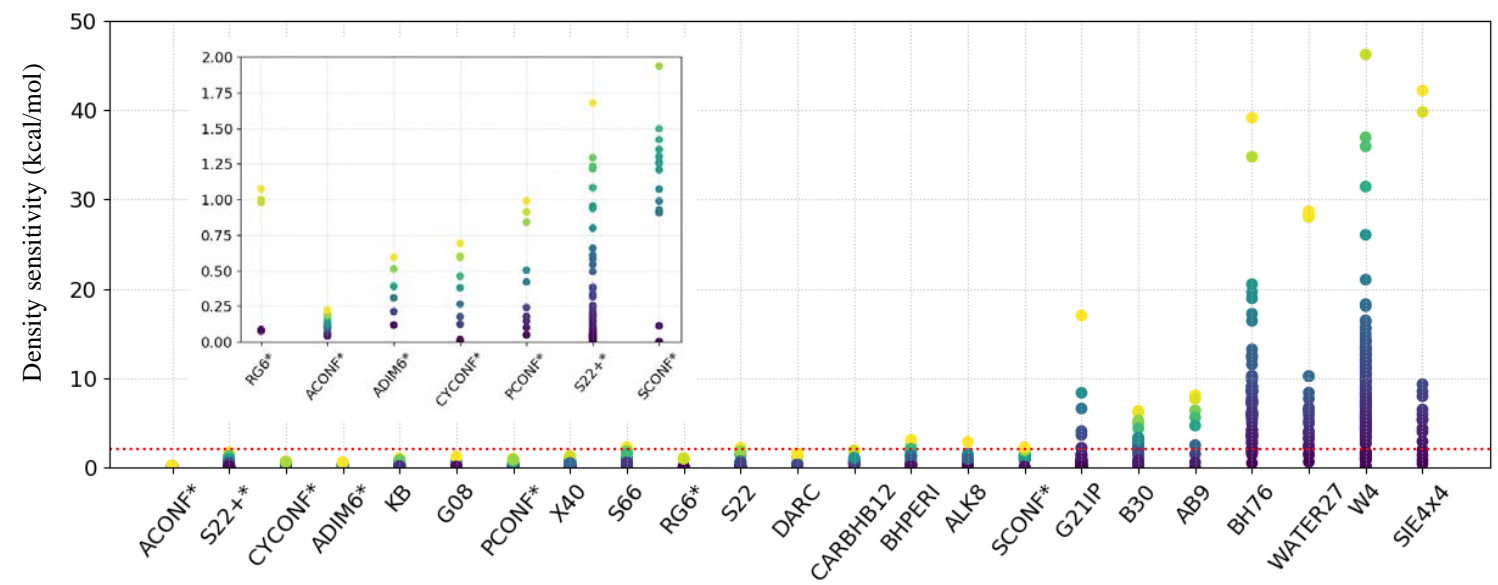

Figure S3: Density sensitivity ( $S^{\mathrm{PBE}}$ ) of PBE for the 23 databases in Table S1. The red dotted line denotes $2 \mathrm{kcal} / \mathrm{mol}$. If $S^{\mathrm{PBE}}$ is greater than $2 \mathrm{kcal} / \mathrm{mol}$, it is considered density sensitive.[19] As we go from the left to the right, the averaged $S^{\mathrm{PBE}}$ ) of the databases increases. The databases used for the training of the Grimme's original D3 parameters are marked with an asterisk. The same databases are also shown in the inset. 


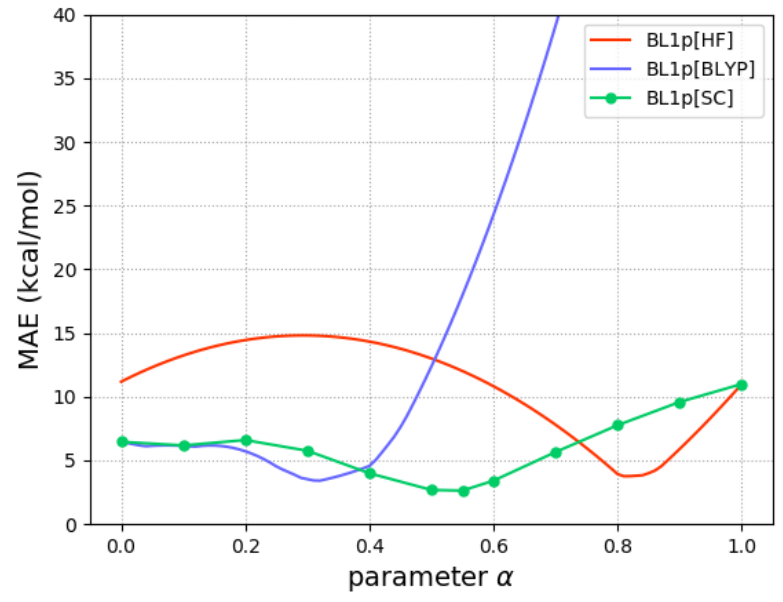

Figure S4: BL1p MAE for the AE6 dataset in Ref. [25]. HF, $B L Y P$ and $S C$ density is used. (SC denotes the self-consistent density from $E_{X C}=\alpha E_{X}^{H F}+(1-\alpha) E_{X}^{B 88}+\left(1-\alpha^{2}\right) E_{C}^{L Y P}$.) Reference values are from Ref. [56].

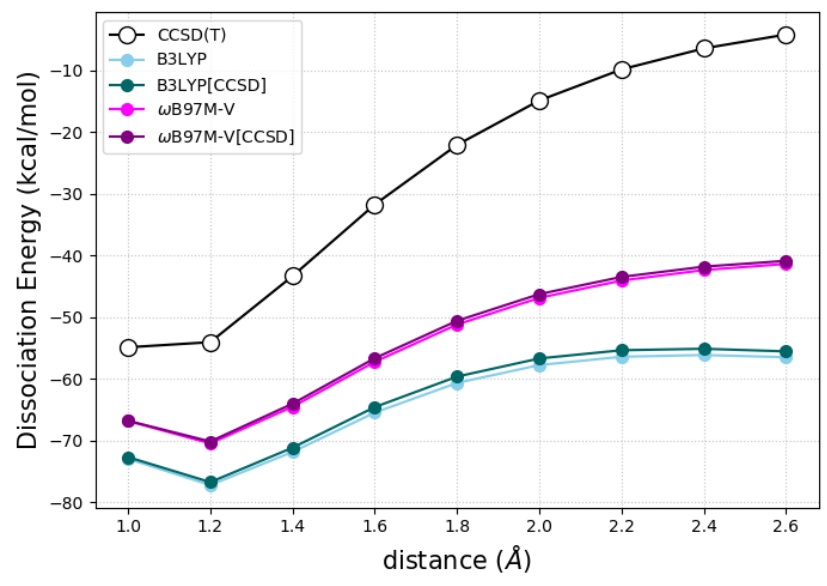

Figure S5: Dissociation curve of $\mathrm{He}_{2}^{+}$. Note that CCSD density is used within the WY KS-inversion method to obtain accurate $K S$ orbitals. Detailed information about KS-inversion can be found in the Ref. [21].

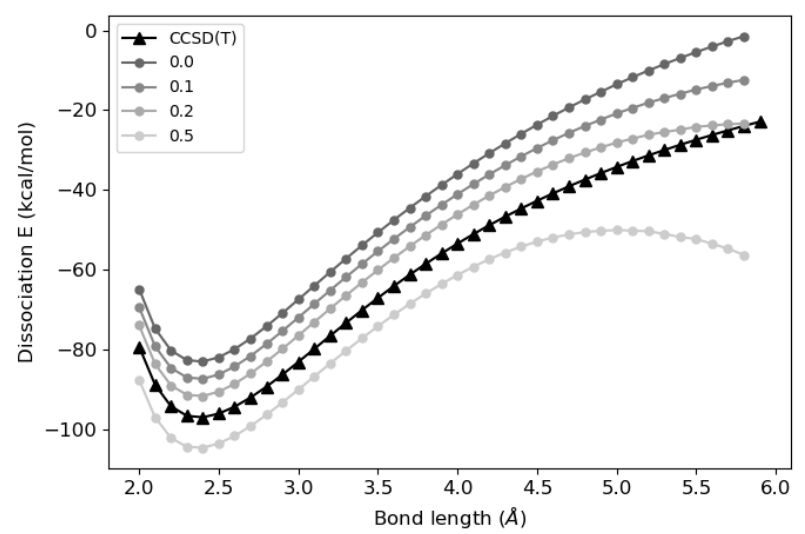

Figure S6: $\mathrm{Na}-\mathrm{Cl}$ dissociation curve for the XYG3 with different $M P 2$ admixture. The larger the MP2 portion, the quicker the XYG3 DH bends down. 


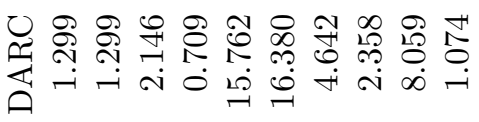

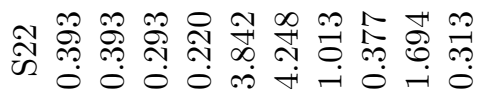

员员员

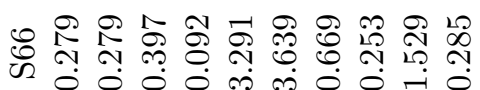

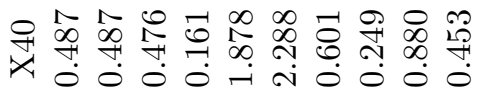

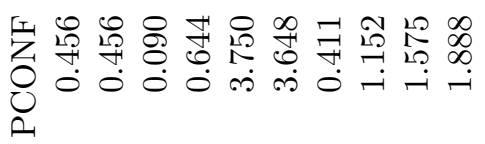

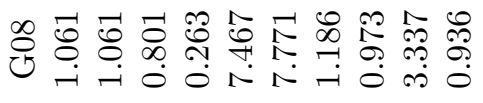

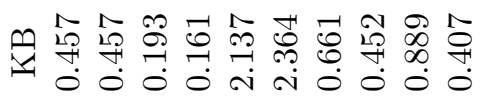

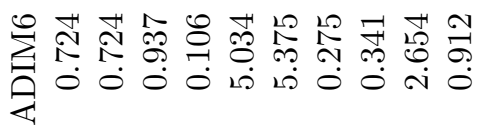

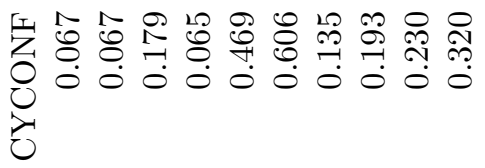

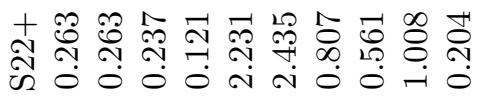

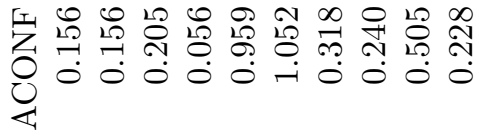

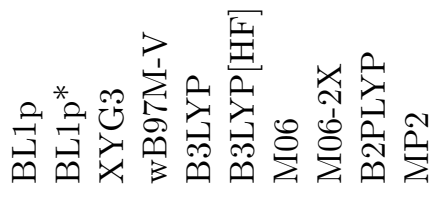

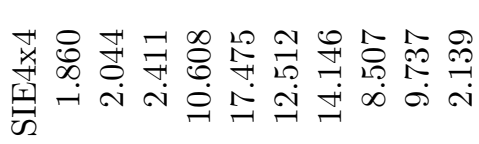

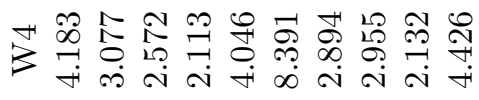

ㅊำ

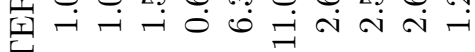

定

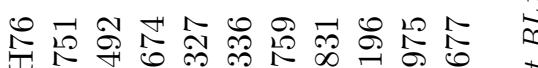

鱼的

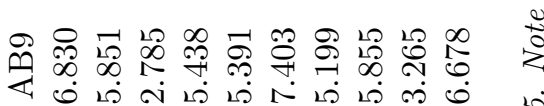

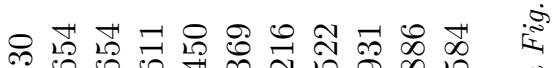

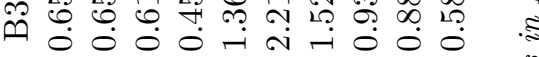

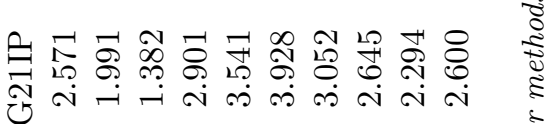

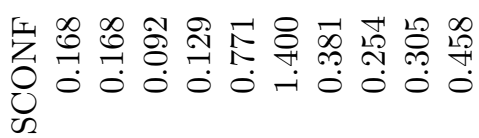

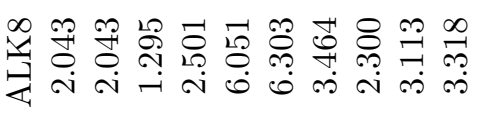

止

利

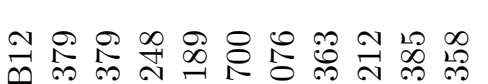

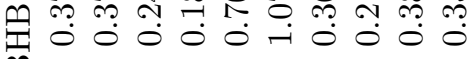

这

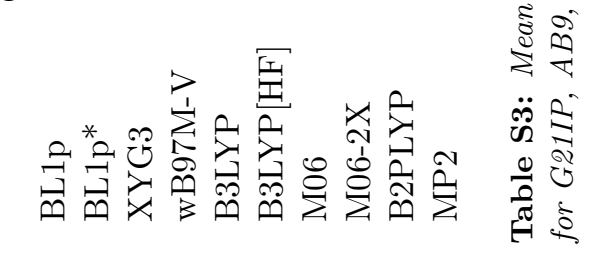




\section{REFERENCES}

[1] Axel D Becke. Density-functional exchange-energy approximation with correct asymptotic behavior. Physical Review A, 38(6):3098-3100, 1988.

[2] Chengteh Lee, Weitao Yang, and Robert G Parr. Development of the colle-salvetti correlation-energy formula into a functional of the electron density. Physical Review B, 37(2):785-789, 1988.

[3] Axel D Becke. Density-functional thermochemistry. iii. the role of exact exchange. The Journal of Chemical Physics, 98(7):5648-5652, 1993.

[4] P. J. Stephens, F. J. Devlin, C. F. Chabalowski, and M. J. Frisch. Ab initio calculation of vibrational absorption and circular dichroism spectra using density functional force fields. The Journal of Physical Chemistry, 98(45):1162311627, 1994.

[5] Haoyu S Yu, Shaohong L Li, and Donald G Truhlar. Perspective: Kohn-sham density functional theory descending a staircase. The Journal of Chemical Physics, 145(13):130901, 2016.

[6] Narbe Mardirossian and Martin Head-Gordon. Thirty years of density functional theory in computational chemistry: an overview and extensive assessment of 200 density functionals. Molecular Physics, 115(19):2315-2372, 2017.

[7] Michael G Medvedev, Ivan S Bushmarinov, Jianwei Sun, John P Perdew, and Konstantin A Lyssenko. Density functional theory is straying from the path toward the exact functional. Science, 355(6320):49-52, 2017.

[8] Enrico Clementi and Subhas J Chakravorty. A comparative study of density functional models to estimate molecular atomization energies. The Journal of Chemical Physics, 93(4):2591-2602, 1990.

[9] Nevin Oliphant and Rodney J Bartlett. A systematic comparison of molecular properties obtained using hartreefock, a hybrid hartree-fock density-functional-theory, and coupled-cluster methods. The Journal of Chemical Physics, 100(9):6550-6561, 1994.

[10] Benjamin G Janesko and Gustavo E Scuseria. Hartreefock orbitals significantly improve the reaction barrier heights predicted by semilocal density functionals. The Journal of Chemical Physics, 128(24):244112, 2008.

[11] David RB Brittain, Ching Yeh Lin, Andrew TB Gilbert, Ekaterina I Izgorodina, Peter MW Gill, and Michelle L Coote. The role of exchange in systematic dft errors for some organic reactions. Physical Chemistry Chemical Physics, 11(8):1138-1142, 2009.
[12] Min-Cheol Kim, Eunji Sim, and Kieron Burke. Communication: Avoiding unbound anions in density functional calculations. The Journal of Chemical Physics, 134(17):171103, 2011.

[13] Prakash Verma, Ajith Perera, and Rodney J. Bartlett. Increasing the applicability of $\mathrm{dft}$ i: Non-variational correlation corrections from hartree-fock $\mathrm{dft}$ for predicting transition states. Chemical Physics Letters, 524:10 - 15, 2012.

[14] Min-Cheol Kim, Eunji Sim, and Kieron Burke. Understanding and reducing errors in density functional calculations. Physical Review Letters, 111(7):073003, 2013.

[15] Min-Cheol Kim, Eunji Sim, and Kieron Burke. Ions in solution: Density corrected density functional theory (dc$\mathrm{dft}$ ). The Journal of Chemical Physics, 140(18):18A528, 2014.

[16] Min-Cheol Kim, Hansol Park, Suyeon Son, Eunji Sim, and Kieron Burke. Improved dft potential energy surfaces via improved densities. The Journal of Physical Chemistry Letters, 6(19):3802-3807, 2015.

[17] Adam Wasserman, Jonathan Nafziger, Kaili Jiang, MinCheol Kim, Eunji Sim, and Kieron Burke. The importance of being self-consistent. Annual Review of Physical Chemistry, 68(1):555-581, 2017.

[18] Suhwan Song, Min-Cheol Kim, Eunji Sim, Anouar Benali, Olle Heinonen, and Kieron Burke. Benchmarks and reliable dft results for spin gaps of small ligand fe (ii) complexes. Journal of Chemical Theory and Computation, 14(5):2304-2311, 2018.

[19] Eunji Sim, Suhwan Song, and Kieron Burke. Quantifying density errors in dft. The Journal of Physical Chemistry Letters, 9(22):6385-6392, 2018.

[20] Yeil Kim, Suhwan Song, Eunji Sim, and Kieron Burke. Halogen and chalcogen binding dominated by densitydriven errors. The Journal of Physical Chemistry Letters, 10(2):295-301, 2018.

[21] Seungsoo Nam, Suhwan Song, Eunji Sim, and Kieron Burke. Measuring density-driven errors using Kohn-Sham inversion. Journal of Chemical Theory and Computation, 16(8):5014-5023, 2020.

[22] Ryo Nagai, Ryosuke Akashi, and Osamu Sugino. Completing density functional theory by machine learning hidden messages from molecules. npj Computational Materials, $6(1): 1-8,2020$.

[23] Stefan Grimme, Jens Antony, Stephan Ehrlich, and Helge Krieg. A consistent and accurate ab initio parametrization of density functional dispersion correction ( $\mathrm{dft}-\mathrm{d}$ ) for the 
94 elements h-pu. The Journal of Chemical Physics, 132(15):154104, 2010.

[24] Stefan Grimme. Semiempirical hybrid density functional with perturbative second-order correlation. The Journal of Chemical Physics, 124(3):034108, 2006.

[25] Kamal Sharkas, Julien Toulouse, and Andreas Savin. Double-hybrid density-functional theory made rigorous. The Journal of Chemical Physics, 134(6):064113, 2011.

[26] Jan ML Martin and Golokesh Santra. Empirical DoubleHybrid Density Functional Theory: A 'Third Way'in Between WFT and DFT. Israel Journal of Chemistry, 60:119, 2019.

[27] Aron J. Cohen, Paula Mori-Sánchez, and Weitao Yang. Insights into current limitations of density functional theory. Science, 321(5890):792-794, 2008.

[28] Aron J Cohen, Paula Mori-Sánchez, and Weitao Yang. Challenges for density functional theory. Chemical Reviews, 112(1):289-320, 2011.

[29] John P Perdew, Kieron Burke, and Matthias Ernzerhof. Generalized gradient approximation made simple. Physical Review Letters, 77(18):3865, 1996.

[30] Stefan Vuckovic, Suhwan Song, John Kozlowski, Eunji Sim, and Kieron Burke. Density functional analysis: The theory of density-corrected dft. Journal of Chemical Theory and Computation, 15(12):6636-6646, 2019.

[31] Eike Caldeweyher, Sebastian Ehlert, Andreas Hansen, Hagen Neugebauer, Sebastian Spicher, Christoph Bannwarth, and Stefan Grimme. A generally applicable atomic-charge dependent london dispersion correction. The Journal of Chemical Physics, 150(15):154122, 2019.

[32] Lars Goerigk, Andreas Hansen, Christoph Bauer, Stephan Ehrlich, Asim Najibi, and Stefan Grimme. A look at the density functional theory zoo with the advanced gmtkn55 database for general main group thermochemistry, kinetics and noncovalent interactions. Physical Chemistry Chemical Physics, 19(48):32184-32215, 2017.

[33] Antonio Bauza, Ibon Alkorta, Antonio Frontera, and Jose Elguero. On the reliability of pure and hybrid $\mathrm{dft}$ methods for the evaluation of halogen, chalcogen, and pnicogen bonds involving anionic and neutral electron donors. Journal of Chemical Theory and Computation, 9(11):5201-5210, 2013.

[34] Narbe Mardirossian and Martin Head-Gordon. wb97mv: A combinatorially optimized, range-separated hybrid, meta-gga density functional with vv10 nonlocal correlation. The Journal of Chemical Physics, 144(21):214110, 2016.
[35] Oleg A. Vydrov and Troy Van Voorhis. Nonlocal van der waals density functional: The simpler the better. The Journal of Chemical Physics, 133(24):244103, December 2010.

[36] Chr. Møller and M. S. Plesset. Note on an approximation treatment for many-electron systems. Physical Review, 46(7):618-622, October 1934.

[37] Benjamin J Lynch and Donald G Truhlar. Small representative benchmarks for thermochemical calculations. The Journal of Physical Chemistry A, 107(42):8996-8999, 2003.

[38] Ying Zhang, Xin Xu, and William A Goddard. Doubly hybrid density functional for accurate descriptions of nonbond interactions, thermochemistry, and thermochemical kinetics. Proceedings of the National Academy of Sciences, 106(13):4963-4968, 2009.

[39] Oleg V Gritsenko and Evert Jan Baerends. Effect of molecular dissociation on the exchange-correlation kohnsham potential. Physical Review A, 54(3):1957, 1996.

[40] Sara Giarrusso, Stefan Vuckovic, and Paola Gori-Giorgi. Response potential in the strong-interaction limit of $\mathrm{dft}$ : Analysis and comparison with the coupling-constant average. Journal of Chemical Theory and Computation, 9(11):3137-3142, 2018.

[41] Oleg A. Vydrov and Gustavo E. Scuseria. Assessment of a long-range corrected hybrid functional. The Journal of Chemical Physics, 125(23):234109, December 2006.

[42] Narbe Mardirossian and Martin Head-Gordon. wb97xv: A 10-parameter, range-separated hybrid, generalized gradient approximation density functional with nonlocal correlation, designed by a survival-of-the-fittest strategy. Physical Chemistry Chemical Physics, 16(21):9904, 2014.

[43] Erin R. Johnson, A. Otero de-la Roza, and Stephen G. Dale. Extreme density-driven delocalization error for a model solvated-electron system. The Journal of Chemical Physics, 139(18):184116, November 2013.

[44] Narbe Mardirossian and Martin Head-Gordon. Mapping the genome of meta-generalized gradient approximation density functionals: The search for b97m-v. The Journal of Chemical Physics, 142(7):074111, February 2015.

[45] Narbe Mardirossian and Martin Head-Gordon. Survival of the most transferable at the top of Jacob's ladder: Defining and testing the $\omega$ B97M (2) double hybrid density functional. The Journal of Chemical Physics, 148(24):241736, 2018.

[46] Golokesh Santra, Nitai Sylvetsky, and Jan M. L. Martin. Minimally empirical double-hybrid functionals trained 
against the GMTKN55 database: revDSD-PBEP86-d4, revDOD-PBE-d4, and DOD-SCAN-d4. The Journal of Physical Chemistry A, 123(24):5129-5143, May 2019.

[47] Michael Seidl, Sara Giarrusso, Stefan Vuckovic, Eduardo Fabiano, and Paola Gori-Giorgi. Communication: Stronginteraction limit of an adiabatic connection in hartree-fock theory. The Journal of Chemical Physics, 149(24):241101, December 2018.

[48] Timothy J. Daas, Juri Grossi, Stefan Vuckovic, Ziad H. Musslimani, Derk P. Kooi, Michael Seidl, Klaas J. H. Giesbertz, and Paola Gori-Giorgi. Large coupling-strength expansion of the møller-plesset adiabatic connection: From paradigmatic cases to variational expressions for the leading terms, 2020.

[49] TURBOMOLE V7.0 2015, a development of University of Karlsruhe and Forschungszentrum Karlsruhe $\mathrm{GmbH}$, 1989-2007, TURBOMOLE GmbH, since 2007; available from http://www.turbomole.com.

[50] Qiming Sun and Timothy C. Berkelbach and Nick S. Blunt and George H. Booth and Sheng Guo and Zhendong $\mathrm{Li}$ and Junzi Liu and James D. McClain and Elvira R. Sayfutyarova and Sandeep Sharma and Sebastian Wouters and Garnet Kin-Lic Chan. PySCF: the Python-based simulations of chemistry framework, 2017.

[51] Paul AM Dirac. Note on exchange phenomena in the thomas atom. In Mathematical Proceedings of the Cambridge Philosophical Society, volume 26, pages 376-385. Cambridge University Press, 1930.

[52] Seymour H Vosko, Leslie Wilk, and Marwan Nusair. Accurate spin-dependent electron liquid correlation energies for local spin density calculations: a critical analysis. Canadian Journal of Physics, 58(8):1200-1211, 1980.

[53] Jianmin Tao, John P Perdew, Viktor N Staroverov, and Gustavo E Scuseria. Climbing the density functional ladder: Nonempirical meta-generalized gradient approximation designed for molecules and solids. Physical Review Letters, 91(14):146401, 2003.

[54] Kieron Burke, Matthias Ernzerhof, and John P Perdew. The adiabatic connection method: a non-empirical hybrid. Chemical Physics Letters, 265:115-120, 1997.

[55] Yan Zhao and Donald G Truhlar. The m06 suite of density functionals for main group thermochemistry, thermochemical kinetics, noncovalent interactions, excited states, and transition elements: two new functionals and systematic testing of four m06-class functionals and 12 other functionals. Theoretical Chemistry Accounts, 120(13):215-241, 2008.
[56] Roberto Peverati and Donald G Truhlar. Improving the accuracy of hybrid meta-GGA density functionals by range separation. The Journal of Physical Chemistry Letters, 2(21):2810-2817, 2011.

[57] http://tccl.yonsei.ac.kr/mediawiki/index. php/DC-DFT. 